
\title{
25 Research Suare \\ Time Trend and Bayesian Mapping of Multiple Myeloma Incidence in Sardinia, Italy
}

\section{Giorgio Broccia}

Hospital A. Businco

Jonathan Carter

University of Coventry

Cansu Ozsin-Ozler

Hacettepe University

\section{Federico Meloni}

University of Cagliari

Ilaria Pilia

University of Cagliari

Sara De Matteis

University of Cagliari

Pierluigi Cocco ( $\nabla$ pcocco@unica.it)

University of Cagliari

\section{Research Article}

Keywords: Multiple myeloma, ecological study, incidence, time trends, Bayesian methods

Posted Date: August 9th, 2021

DOI: https://doi.org/10.21203/rs.3.rs-702196/v1

License: (c) (i) This work is licensed under a Creative Commons Attribution 4.0 International License.

Read Full License

Version of Record: A version of this preprint was published at Scientific Reports on February 17th, 2022. See the published version at https://doi.org/10.1038/s41598-022-06745-z. 


\section{Abstract}

A few reports have described increasing trends and spatial distribution of multiple myeloma (MM). We used a validated database including the 1606 cases of MM diagnosed in Sardinia in 1974-2003 to explore its time trend, and we applied Bayesian methods to plot MM probability by administrative unit on the regional map. Over the 30 years of observation, the MM standardized incidence rate (standard world population, all ages) was $2.17 \times 10^{-5}$ (95\% Cl 2.01-2.34), 2.29 (95\% $\left.\mathrm{Cl} 2.06-2.52\right)$ among men, and 2.06 (95\% $\mathrm{Cl} 1.83-2.28)$ among women. MM incidence increased by 3.3\%/year in 1974-2003, in both males and females, particularly among the elderly and in the high incidence areas. Areas at risk tended to cluster in the north-eastern part of the region. A higher proportion of elderly in the resident population, but not socioeconomic factors, nor livestock farming, was associated with higher incidence rates. The steep upward time trend and the spatial clustering of MM suggest interactions between genetic and environmental determinants that might be more efficiently investigated in the areas at risk.

\section{Introduction}

Multiple myeloma $(\mathrm{MM})$ is a mature $B$ cell lymphoma, which worldwide standardized rate is $2.1 \times 10^{-5}$ (95\% $\mathrm{Cl} 1.8-2.3)$ [1], ranging from $0.1-4.5$ among the male population of Asian countries to $10.2-13.1 \mathrm{x}$ $10^{-5}$ among the male Afro-American population of Texas and Wisconsin [2]. MM incidence is more elevated among men; increases sharply with age [3]; and it occurs more frequently among farmers and following exposure to ionizing radiation, certain pesticides, and solvents [4]. However, its etiology is still unclear.

As it concerns its time and space variability, an increasing time trend of $\mathrm{MM}$ has been described in the Czech Republic [5], South America [6], Taiwan [7], and in Canada [8]. Three studies described an excess risk in metropolitan and urban areas $[6,8,9]$, while no spatial clustering of $M M$ cases was observed in a UK study [10].

The Sardinian population is well known for its peculiar genetic features [11], which have provided the ideal setting for studies on the genes associated with the local high prevalence of male centenarians [12], multiple sclerosis [13], or type I childhood diabetes. The study of spatial distribution of MM incidence over the island might provide clues for future more in depth studies on genetic susceptibility and geneenvironment interactions in MM aetiology.

Based on the data reported in the 11th Edition of the International Agency for Research on Cancer (IARC) Cancer Incidence in the Five Continents (Cl5-11), the incidence rate ranges 2.6-5.4 $\times 10^{-5}$ across the 33 Italian Cancer Registries [2]. The history of cancer registration in Sardinia is relatively recent, fragmented, and discontinuing: the Sassari Cancer Registry, covering the northern part of the island and about $30 \%$ of the resident population, started in 1993 and ceased operating in 2013; the Nuoro Cancer Registry, covering the central-eastern part of the island and $13 \%$ of the total population, started in 2003 and it keeps functioning. After several decades of unsuccessful attempts, a Cancer Registry has been planned 
for southern Sardinia, but it might take an unpredictable length of time to start operating regularly. The standardized incidence (world population, all ages) reported in the $\mathrm{Cl} 5-11$ is 2.9 among males and $2.8 \mathrm{x}$ $10^{-5}$ among females in northern Sardinia, and 4.0 among males and 2.1 among females in its centraleastern part [2].

To get around the lack of an official system of regular surveillance of cancer incidence, in 1974, the chief haematologist of the Cagliari Oncology Hospital (BG) initiated a database of incident cases of haemolymphatic malignancies over the whole region, with the collaboration of all the clinical, surgical and pathology departments, social security agencies, and health authorities, and he kept updating it up to 2003, as described elsewhere [14]. To explore the time trend and the geographical distribution of multiple myeloma over the territory of the island, we used such database, which was previously validated by comparison with mortality and hospitalization data [15].

\section{Results}

\section{Time trend in MM incidence}

In 1974-2003, 1606 MM cases occurred among the Sardinian population. Based on the standard World population, the incidence rate over these three decades was $2.17 \times 10^{-5}(95 \% \mathrm{Cl} 2.01-2.34)$ for the total population (all ages), 2.29 (95\% Cl 2.06-2.52) among males, and 2.06 (95\% Cl 1.83-2.28) among females, with a male/female ratio of 0.95 . The crude rate over the study period was $2.73 \times 10^{-5}(95 \% \mathrm{Cl}$ 2.57-2.89) among the total population, 3.17 (95\% Cl 2.94-3.40) among males, and $2.30(95 \% \mathrm{Cl} 2.07-$ 2.53) among females. Graphs in Fig. 1 show the trend of MM incidence, by gender: on average, MM incidence increased linearly from 1974 through 2003 by $0.124 \times 10^{-5}$ per year (3.3\%/year) $\left(p=4.76 \times 10^{-}\right.$ $\left.{ }^{11}\right)$. The upward trend was similar among the female $\left(0.189 \times 10^{-5}\right.$ per year, $\left.p=0.007\right)$ and the male population $\left(0.113 \times 10^{-5}\right.$ per year, $\left.p=2.02 \times 10^{-7}\right)$, with a slope 10 times steeper above the age of 65 $\left(0.479 \times 10^{-5}\right.$ per year, $\left.p=3.49 \times 10^{-7}\right)$ than below $65\left(0.049 \times 10^{-5}\right.$ per year, $\left.p=6.14 \times 10^{-9}\right)$ (analysis of covariance: $\left.\mathrm{F}=307.37, \mathrm{DF}=58 ; p=1.25 \times 10^{-24}\right)$. The upward time trend was observed in the areas with incidence rate below the median $\left(0.110 \times 10^{-5}\right.$ per year, $\left.p=1.9 \times 10^{-12}\right)$, as well as in the areas where incidence rate was above the median $\left(0.207 \times 10^{-5}\right.$ per year, $\left.p=1.39 \times 10^{-5}\right)$, but its slope was twice as steep in the high incidence areas (analysis of covariance: $\mathrm{F}=61.5, \mathrm{DF}=58 ; p=5.29 \times 10^{-11}$ ). A comparison with cancer registry data was possible for a limited area in the last decade of the time span covered by the database we used. In 1993-1997, the Sassari Cancer Registry data reported a MM incidence, based on the world standard population, of $2.7 \times 10^{-5}$ among men and 2.1 among women [16], which increased up to 3.5 among men and 2.9 in 1998-2002 [17]. The corresponding figures for the same area in our data base were 2.4 for men and 1.7 for women in 1993-1997, and 2.8 for men and 2.1 for women in 1998-2002. In 2008-2012, MM incidence (world standard population) was stable for both genders in the northern part of the region (Sassari Cancer Registry: men $2.9 \times 10^{-5}$; women $2.8 \times 10^{-5}$ ), while it was $4.0 \times 10^{-5}$ among men, more than double the 1974-2003 figure from the clinical database 
$\left(1.8 \times 10^{-5}\right)$, and $2.1 \times 10^{-5}$ among women, substantially similar to the $1974-2003$ incidence $\left(2.3 \times 10^{-}\right.$ $5)$, in the Nuoro Cancer Registry [2].

\section{Geographic map of MM incidence}

Figure 2 shows the map of the probability of a MM incidence rate above the critical rate in each of the 356 Sardinian communes, overall and by gender. Seven communes stand out exceeding the 95th percentile distribution of the likelihood ratio. These are: Arborea (9 cases, likelihood ratio 20.2, $p=0.953$ ), Padria (5 cases, likelihood ratio 20.2, $p=0.953$ ), Benetutti (8 cases, likelihood ratio 46.8, $p=0.979$ ), Bitti (11 cases, likelihood ratio 59.2, $p=0.983$ ), Oschiri (5 cases, likelihood ratio 70.0, $p=0.986$ ), Perfugas (10 cases, likelihood ratio 75.2, $p=0.987$ ), and Seneghe (9 cases, likelihood ratio 177.9, $p=0.994)$. Another 25 communes have a probability ranging $80-94 \%$, based on 3 or more cases. The map shows a tendency of high incidence communes to concentrate in the north-eastern area of the island, with the low incidence areas located in the southern areas and in the two major urban areas, one, Cagliari, in the south, and the second, Sassari, in northwest Sardinia.

\section{Analysis of MM risk factors}

We explored with weighted multiple regression analysis whether socioeconomic conditions, or size of livestock, or an aging population might have generated the wide geographic variability in MM incidence we observed. As an indicator of socioeconomic conditions, we used the Italian National Institute of Statistics (ISTAT) deprivation index, which combines the following: proportion of the resident population who attained elementary education at most; proportion of the resident population aged 15 years or older searching for an occupation; proportion of the active population engaged in manual work; proportion of rentals over the total residences; persons per room in the household [18]. Independent covariates were male/female ratio of the resident population, deprivation index, presence of large livestock farms (cattle, sheep, and goats) [19], and proportion of the population aged $\geq 65$ years. We used the commune population sizes relative to the overall regional population as the weights. The only covariate showing a significant association with $\mathrm{MM}$ incidence was the proportion of elderly among the resident population $\left(\beta=0.609\right.$, se $\left.=0.085 ; p=7.8 \times 10^{-13}\right)$.

\section{Discussion}

Our results show that in 1974-2003 MM incidence increased in both genders among the Sardinian population. Cancer Registry data confirmed such finding limited to the northern area of the region and the last decade covered by the database we used. There was no further increase in the subsequent years. The steeper slope of the regression line among the elderly, and the observed association between proportion of elderly among the resident population and MM incidence, suggest that the increasing aging of the Sardinian population and the increasing access of the elderly to specialized medical care over the study period might have contributed to a more frequent diagnosis of the disease along the years. However, the same upward time trend was also observed among the population aged $<65$, indicating that other factors 
might have played a role, possibly interacting with genetic susceptibility to generate the steeper upward time trend we observed in high-risk areas.

Seven communes stand out with the highest probability of their rate exceeding that of the overall population of the region, four located in the northwestern area (Benetutti, Bitti, Oschiri, and Perfugas) and three scattered in the north west and central areas. These are mainly agricultural areas, six with archeological remains indicating their origin dating back to prehistory, and one, Arborea, built in 1928 over a reclaiming land, previously covered by marshes in a malaria endemic area. Most of the population hosted in this new city came in the early years of its foundation from Veneto and Friuli, two regions in north east Italy, and created a flourishing livestock and agricultural crop economy. This town is also known for the large size of its livestock, with two thirds of Sardinian cattle raised in its land. However, we did not find a relationship between presence of large cattle farms and MM incidence over the whole region.

The finding of an excess risk associated with having a first-degree relative affected by MM, particularly among men, and African Americans, supports a role of genetic factors [20]. On the other hand, about 17\% of MM heritability seems explained by the known gene variants [21]. Besides, based on results from molecular biology studies, aberrant class switch recombination occurring early in the natural history of MM suggests that environmental factors, such as high doses of ionizing radiation, and occupational exposure in the farming and petrochemical industries, might also contribute to increase risk [22]. The DNA damage resulting from environmental exposures would interact with the class switch recombination process to increase the risk of chromosomal translocations, oncogene deregulation, and malignant transformation [22]. In an analysis of MM risk related to occupation, a moderate increase in risk was reported in association with contact with livestock [23]. Also, gardeners and nursery workers combined, but not other farming jobs, metal processors, female cleaners, and occupations with high level exposure to organic solvents showed a moderate increase in risk [24]. Among lifestyle factors, a moderate alcohol intake might would reportedly convey protection [25].

Although Sardinia is well known for the genetic peculiarities of its population [11], we are not aware of genetic investigations aiming to identify the varying prevalence of gene polymorphisms implicated in MM. The small town of Arborea, with his peculiar modernist architecture, is home for about 4,000 inhabitants, a large fraction of whom preserved their original language, diet, and habits. This population has a different ethnic origin than the rest of the Sardinian population, but it is unclear whether this might be related to the excess incidence of MM therein observed. Nonetheless, the incidence for the resident population (both genders), standardized based on the world population, was $4.6 \times 10^{-5}, 5.05$ among men, and 4.2 among women. The corresponding rates in the Veneto region cancer registry were 4.5 for men and 3.7 for women in the IARC CI5 10th Edition [26], and 4.1 for men and 2.7 for women in its 11th Edition [2]. The corresponding figures in the Friuli Cancer Registry were 3.8 for men and 2.9 for women [26], and 3.3 for men and 2.5 for women [2], consistent with what observed in the small city of Arborea in 1974-2003. 
The lack of exact correspondence with Cancer Registry data might be due to the fact that the database we used include the cases resulting from final clinical and pathological work up, i.e. those who accessed a dedicated haematology unit that could perform the necessary tests, such as platelets count, light chains in serum and urine, bone marrow aspiration execution and evaluation, bone marrow biopsy, and cytochemistry. These might not include all the incident cases, also because of co-occurrence of MM with other diseases among the elderly, which might result in underdiagnosis. However, this would affect mostly small villages far apart from the specialized haematology units, located in the major urban centres; however, the elevated risk was mainly observed in small towns, which would contrast this hypothesis.

For the same reason, post-diagnosis relocation of the families seems unlikely to have occurred. The exact address at the time of diagnosis was missing for $58 / 1606$ patients (3.6\%); it seems also unlikely that this might have affected the overall pattern.

An advantage of our study is that the diagnoses were all reviewed by the same expert haematologist (GB), thus preventing bias due to the varying diagnostic ability by time and geographic area and minimizing and spreading equally the probability of misdiagnosis over the whole region and along the study period. The substantial similarity between the incidence data calculated from our database, and those from Cancer Registry data, although limited to the last decade covered by the clinical database we used and to the northern part of the island, supports the completeness of its records.

\section{Conclusion}

Our results describe for the first time increasing time trends of multiple myeloma over several decades among the population of the island of Sardinia. Multiple myeloma incidence increased in both genders, and particularly among the elderly and in high-risk areas. We also observed a clustering of high MM incidence in the north-eastern area, which might be of interest for future gene-environment interaction studies. Finally, our results might support the extension of the Cancer Registry coverage to the whole Sardinian population, which would be of paramount importance not only for fostering further research, but also for early detection of risk areas, so to promote effective preventive intervention, and for a rational planning of cancer treatment resources.

\section{Material And Methods}

A detailed description of the database of haemolymphatic malignancies we used in this study can be found elsewhere [14]. Briefly, it includes 14,744 incident cases of any haematological cancer, in both genders, and at any age diagnosed in the Italian region of Sardinia in 1974-2003. For the purposes of this analysis, we selected the 1,606 MM cases, 781 males and 825 females.

For each commune (the smallest administrative unit in Italy), we calculated the total person-years for each gender and age group $(0-24,25-34,35-44,45-54,55-64,65-74$, and $74+)$ over the study period, 
from January 1974 through December 2003. The standardized incidence rate, annual and over the whole study period, of MM was calculated using the 1971, 1981, 1991, and 2001 census data of the regional population as the standard. Census figures were extended four years onwards and five years backwards to estimate the resident population in the intercensal years. Incidence rates were plotted against time to calculate the regression equation and the Pearson's correlation coefficient. To compare results with those from the IARC $\mathrm{Cl} 5$ volumes, we also standardized the regional, gender specific incidence rates using the standard world population. We used analysis of covariance to test the chance probability associated with the different slope of regression coefficients by age at diagnosis (below or above 65 years old), and by residence in an area with low $v s$ high probability of MM occurrence, using the median as the cut point [27].

The statistics to explore the spatial distribution of the probability of MM occurrence have been previously described in detail [28]. Briefly, we used a Bayesian approach, which allowed us to combine information on $\mathrm{MM}$ incidence over the island with that from the individual communes with the following equation:

$$
P(\eta \mid d, I)=\frac{P(d \mid \eta, I) P(\eta \mid I)}{P(d \mid I)}
$$

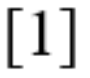

where $P(\eta / d, l)$ is the posterior probability distribution of MM incidence rate $\eta$ for a given commune, after combining the data $d$ for that commune with those from the whole region. $P(\eta / I)$ is the prior MM standardized incidence rate, $\eta$, from the background regional information; $P(d / \eta, I)$ is the probability of getting $d$ for the commune assuming $\eta$ is true, and $P(d / I)$ sets to one the integral of the posterior probability $P(\eta / d, I)$ over all possible values of $\eta$, so to obtain a probability density function.

To detect communes at high risk, we set the critical value of $p=0.001$ in the prior probability distribution of MM incidence over the 356 Sardinian communes, and, for each commune and each gender and age subgroup, we calculated the likelihood ratio between the probability of a MM incidence rate higher than the critical probability level and that of a MM incidence rate consistent with what observed at the regional level.

Finally, we plotted on the regional map the probability associated with the likelihood ratio for each commune, using the following colour scale for the area of each commune, based on the quintiles of the probability distribution: white $<0.165$, light grey $0.166-0.335$, medium-light grey $0.336-0.50$, medium-dark grey $0.501-0.80$, dark grey $0.801-0.95$. The few communes associated with a probability higher than $95 \%$ had the darkest black shade.

The methods used in this study do comply with the requirements for ecological studies, including the acknowledgement of the limitations of such study design, as highlighted by Rezaeian's call for a still missing STROBE statement on ecological studies [29]. Our ecological study was based on the analysis of aggregated data; no humans were involved. Therefore, the informed consent requirement for participation does not apply. The use of these aggregated data for the purposes of scientific publication was approved 
by the Ethics Committee of the University Hospital of Cagliari (protocol N. PG 2019/18070, 18 December 2019).

\section{Declarations}

\section{Data availability:}

data are preserved in the archives of the Department of Medical Sciences and Public Health of the Cagliari University in aggregated form, and they are publicly available as such. Please contact Prof. Pierluigi Cocco (pcocco@unica.it) for any request.

\section{Funding.}

This research did not receive any grant from funding agencies in the public, commercial, or not-for-profit sectors.

\section{Authors' contributions:}

G.B.: Conceptualization, Data curation, Validation, Project administration, Writing - review \& editing. J.C.: Conceptualization, Methodology, Formal analysis, Roles/Writing - original draft, Writing - review \& editing. C.O.-O.: Visualization, Writing - review \& editing. F.M.: Formal analysis, Visualization, Writing - review \& editing. I. P.: Formal analysis, Visualization, Writing - review \& editing. S.DeM.: Supervision, Writing review \& editing. P.C.: Conceptualization, Methodology, Supervision, Roles/Writing - original draft, Writing review \& editing.

\section{Competing interests.}

All the authors declare no competing interests.

\section{References}

1. Cowan, A.J., et al. Global Burden of Multiple Myeloma: A Systematic Analysis for the Global Burden of Disease Study 2016. JAMA Oncol. 4, 1221-1227 (2018). https://doi:

10.1001/jamaoncol.2018.2128.

2. Cavalli-Sforza, L. L., Menozzi, P. \& Piazza A The History and Geography of Human Gene. J. $R$. Anthropol. Inst, 2, 413 https://doi.org/https://doi:10.2307/3034645 (1996).

3. Francalacci, P. et al. Low-pass DNA sequencing of 1200 Sardinians reconstructs European Ychromosome phylogeny., 341, 565-569 https://doi.org/10.1126/science (2013). https://doi 
4. Fazia, T. et al. Investigating multiple sclerosis genetic susceptibility on the founder population of east-central Sardinia via association and linkage analysis of immune-related loci. Mult. Scler, 24, 1815-1824 https://doi.org/https://doi:10.1177/13524585 17732841 (2018).

5. Broccia, G. et al. A 30-year epidemiologic survey (1974-2003) of haematological malignancies on the island of Sardinia: temporal changes in incidence and a geographic comparison of incidence rates. Ann. Hematol, 93, 1041-1049 https://doi.org/https://doi:10.1007/ s00277-013-2002-z (2014).

6. Cocco, P. [Lessons learned from the 'Quirra syndrome' epidemiology? No, thanks]. Epidemiol. Prev, 36, 41-44 (2012).

7. International Agency for Research on Cancer. Cancer Incidence in Five Continents, Vol. VIII. (eds. Parkin, D.M., Whelan, S.L., Ferlay, J., Teppo, L. \& Thomas, D.B.)93-771(IARC, 2002).

8. International Agency for Research on Cancer Cancer Incidence in Five Continents, Vol. IX. (eds. Curado, M.P., et al.)417-893(IARC, 2007).

9. Minerba, L. \& Vacca, D. [Deprivation indexes for the analysis of inequality across the communes of Sardinia]. In: https://istat.it/it/archivio/6727 (2006).

10. LAORE - Agenzia Regionale per lo Sviluppo in Agricoltura [Data on cattle, sheep, and goat farms in Sardinia - 2019]. http://sardegnaagricoltura.it/documenti (2020).

11. Schinasi, L. H. et al. Multiple myeloma and family history of lymphohaematopoietic cancers: results from the International Multiple Myeloma Consortium. Br. J. Haematol, 175, 87-101 https://doi.org/10.1111/bjh.14199 (2016). https://doi

12. Chattopadhyay, S. et al. Genome-wide interaction and pathway-based identification of key regulators in multiple myeloma. Commun. Biol, 2, 89 https://doi.org/https://doi:10.1038/ s42003-019-0329-2 (2019).

13. Morgan, G. J., Davies, F. E. \& Linet, M. Myeloma aetiology and epidemiology. Biomed. Pharmacother, 56 (5), 223-234 https://doi.org/https://doi:10.1016/s0753-3322(02)00194-4 (2002).

14. Svec, M. A., Ward, M. H., Dosemeci, M., Checkoway, H. \& De Roos, A. J. Risk of lymphatic or haematopoietic cancer mortality with occupational exposure to animals or the public. Occup. Environ. Med, 62, 726-735 https://doi.org/https://doi:10.1136/oem.2005 (2005).

15. Perrotta, C. et al. Multiple myeloma and occupation: a pooled analysis by the International Multiple Myeloma Consortium. Cancer Epidemiol, 37, 300-305 https://doi.org/https://doi:10.1016/j.canep.2013.01.008 (2013).

16. Andreotti, G. et al. A pooled analysis of alcohol consumption and risk of multiple myeloma in the international multiple myeloma consortium. Cancer Epidemiol. Biomarkers Prev, 22, 1620-1627 https://doi.org/https://doi:10.1158/1055-9965.EPI-14-1145 (2013).

17. International Agency for Research on Cancer. Cancer Incidence in Five Continents, Vol. X. (eds. Forman, D., et al)126-1363(IARC, 2013).

18. Snedecor, G. W. \& Cochran, W. G. Statistical methods 6th edn (The lowa State University Press, 1967). 
19. Broccia, G. et al. Haemolymphatic cancer among children in Sardinia, Italy: 1974-2003 incidence. BMJ Open, 10, e037163 https://doi.org/https://doi:10.1136/bmjopen-2020-037163 (2020).

20. Rezaeian, M. A call for revising the strengthening the reporting of observational studies in epidemiology statement to include ecologic studies. J. Clin. Epidemiol, 67, 836 https://doi.org/https://doi: 10.1016/j.jclinepi.2014.02.010 (2014).

21. 21. Chattopadhyay, S., et al. Genome-wide interaction and pathway-based identification of key regulators in multiple myeloma. Commun. Biol. 2: 89 (2019). https://doi:10.1038/ s42003-019-03292

22. 22. Morgan, G.J., Davies, F.E., Linet, M. Myeloma aetiology and epidemiology. Biomed. Pharmacother. 56(5): 223-234 (2002). https://doi:10.1016/s0753-3322(02)00194-4.

23. 23. Svec, M.A., Ward, M.H., Dosemeci, M., Checkoway, H., De Roos, A.J. Risk of lymphatic or haematopoietic cancer mortality with occupational exposure to animals or the public. Occup. Environ. Med. 62:726-735 (2005). https://doi:10.1136/oem.2005. 021550.

24. 24. Perrotta, C., et al. Multiple myeloma and occupation: a pooled analysis by the International Multiple Myeloma Consortium. Cancer Epidemiol. 37: 300-305 (2013). https://doi:10.1016/j.canep.2013.01.008.

25. 25. Andreotti, G., et al. A pooled analysis of alcohol consumption and risk of multiple myeloma in the international multiple myeloma consortium. Cancer Epidemiol. Biomarkers Prev. 22: 1620-1627 (2013). https://doi:10.1158/1055-9965.EPI-14-1145.

26. 26. International Agency for Research on Cancer. Cancer Incidence in Five Continents, Vol. X. (eds. Forman, D., et al) 126-1363 (IARC, 2013).

27. 27. Snedecor, G.W. \& Cochran, W.G. Statistical methods. 6th ed. (The lowa State University Press, 1967).

28. 28. Broccia, G., et al. Haemolymphatic cancer among children in Sardinia, Italy: 1974-2003 incidence. BMJ Open 10: e037163 (2020). https://doi:10.1136/bmjopen-2020-037163.

29. 29. Rezaeian, M. A call for revising the strengthening the reporting of observational studies in epidemiology statement to include ecologic studies. J. Clin. Epidemiol. 67: 836 (2014). https://doi: 10.1016/j.jclinepi.2014.02.010.

\section{Figures}


a. Males

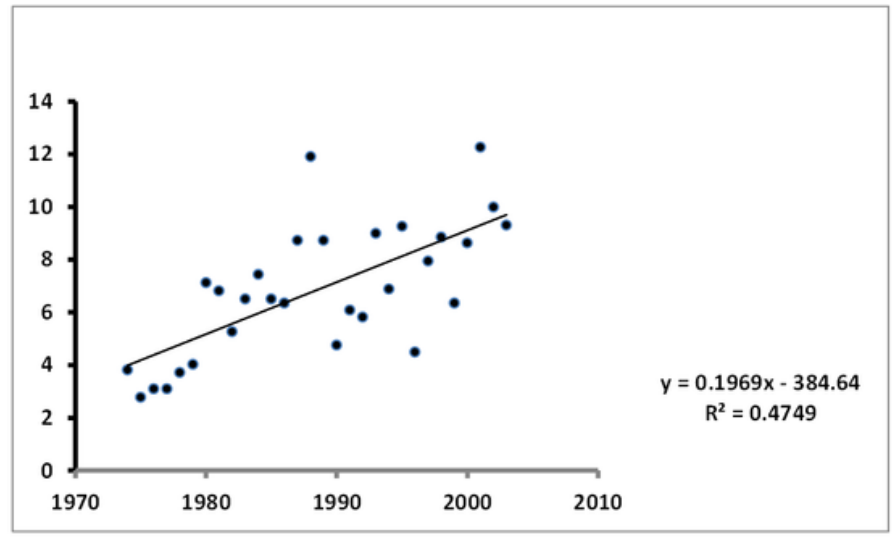

b. Females

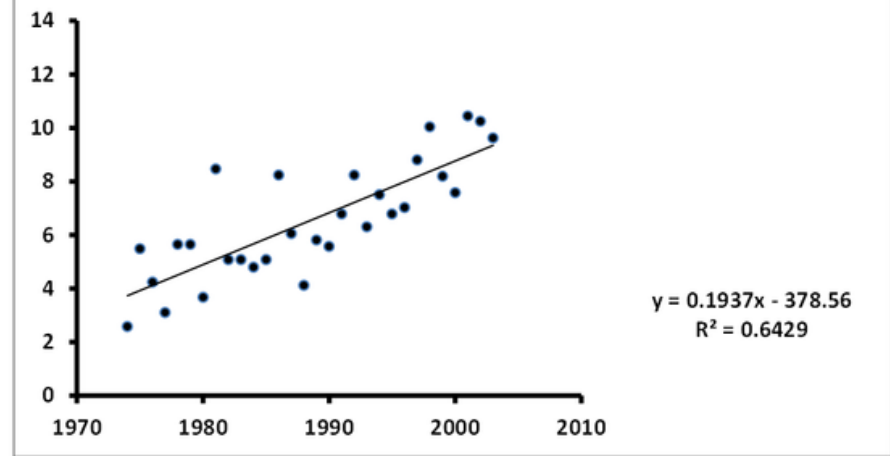

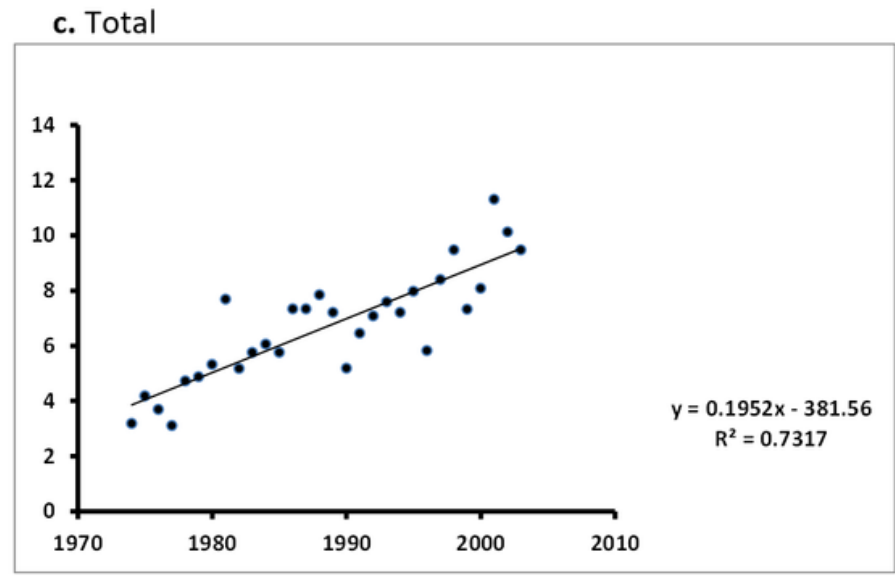

Figure 1

1974-2003 annual incidence rate of multiple myeloma in Sardinia, Italy, by gender (a, b) and overall (c). 
a.

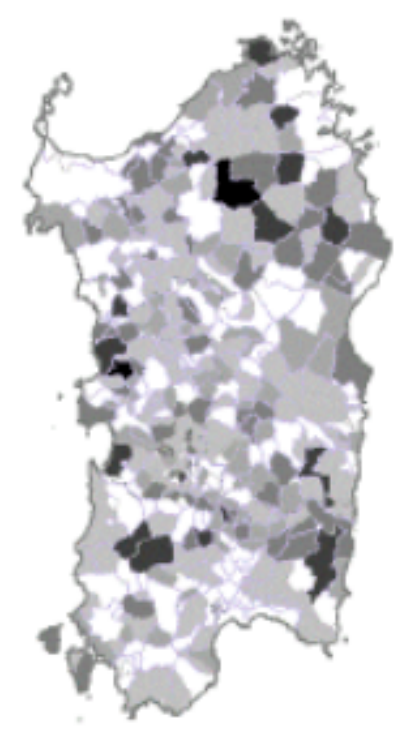

b.

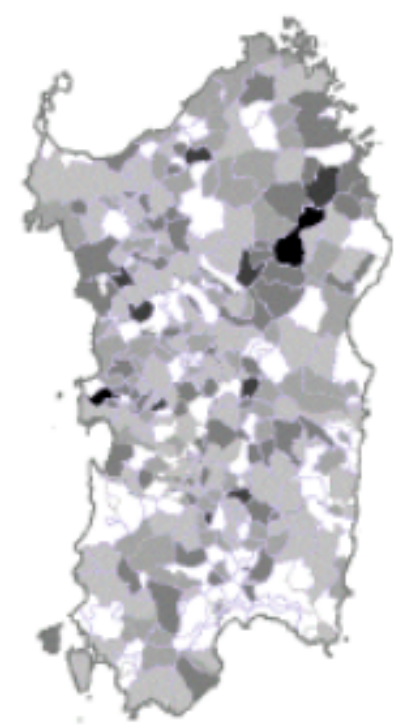

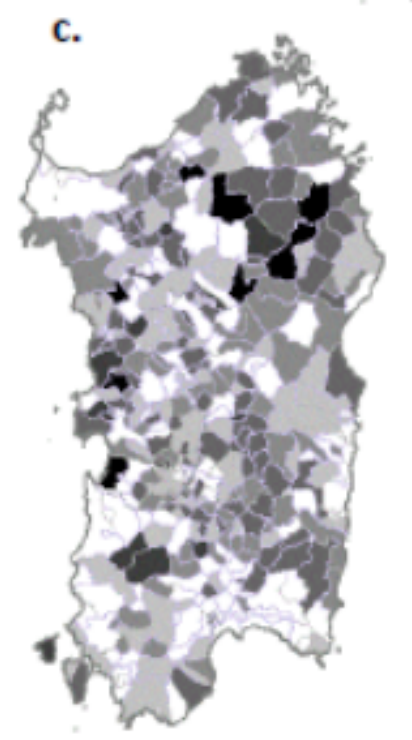

Figure 2

Map of multiple myeloma incidence in Sardinia, Italy. Likelihood ratios are presented by commune with the following color scales: white $<0.165$, pale grey $0.166-0.335$, pale-medium grey $0.336-0.50$, mediumdark grey $0.501-0.80$, dark grey $0.801-0.95$, black $>0.951$. a: males; b: females; c: total population. 